\title{
Old habits die hard
}

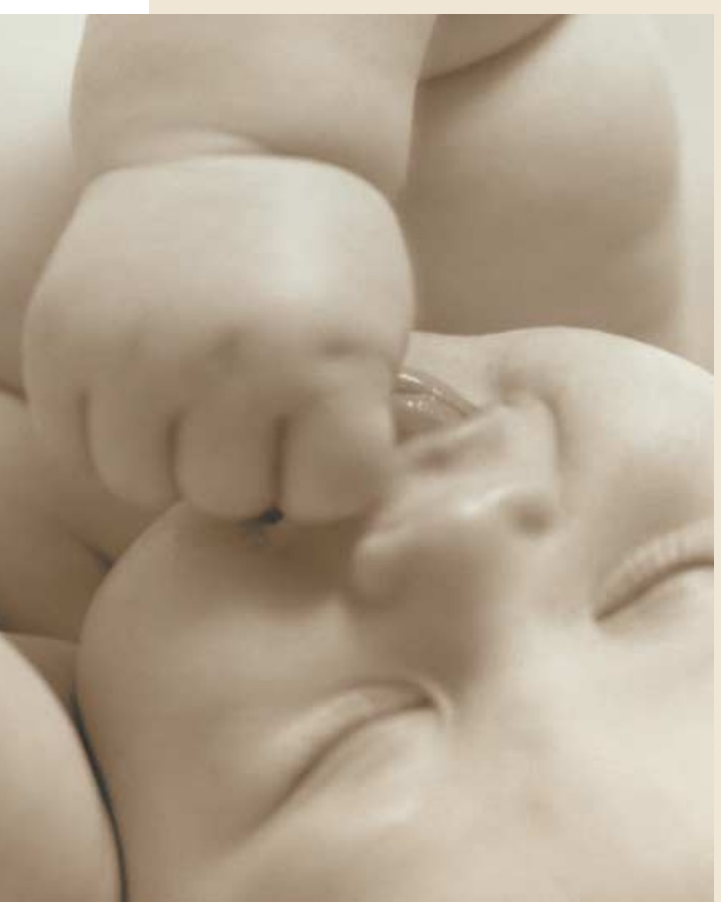

Habit formation has been proposed to depend on the dorsolateral striatum (DLS), but direct evidence for this has been scarce. An elegant study published in the European Journal of Neuroscience now shows that lesions of the DLS disrupt habit formation but preserve outcome expectancy in rats.

Yin et al. trained control and DLS-lesioned rats to press a lever to obtain sucrose using a training protocol known to turn this action into a habit. After training, the experimenters changed the value of the reward in half of the animals in each group by pairing the sucrose with injections of lithium chloride, inducing conditioned taste aversion. As expected, all the animals that received lithium chloride learned to avoid the taste, as this form of learning does not depend on the DLS. The key finding came from a subsequent test in which the rats were re-exposed to the lever. Control rats ceased to consume the sucrose, but continued to press the lever; performance of this action had become insensitive to changes in the value of the outcome, a hallmark of habit formation. By contrast, DLS-lesioned rats showed a marked reduction in lever pressing; performance was sensitive once more to its outcome, which was now devalued after the induction of taste aversion.

These results show a compelling dissociation between habit formation and outcome expectancy. Furthermore, they provide evidence that when the habitformation system is impaired, behaviour is dominated by the system that governs the formation of response-outcome associations. Future studies should aim to disentangle the way in which the two systems interact to control instrumental conditioning.

(4) References and links

Juan Carlos López

ORIGINAL RESEARCH PAPER Yin, $H$. H. et al. Lesions of dorsolateral striatum preserve outcome expectancy but disrupt habit formation in instrumental learning. Eur. J. Neurosci. 19, 181-189 (2004)

\section{Look deep into my brain...}

The idea that a microscope can be used to study the cortex in living, behaving animals is amazing, but true. However, even the most powerful multiphoton techniques are currently limited to a few hundred micrometres below the surface of the brain. Levene et al., writing in The Journal of Neurophysiology,

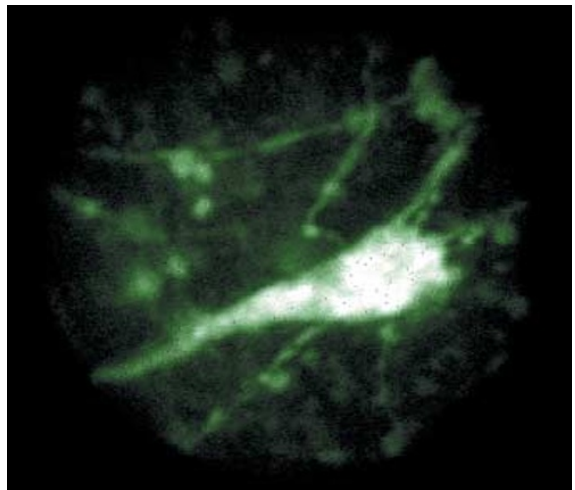

Microscopic image of a layer $\vee$ neuron $~ 750 \mu \mathrm{m}$ below the surface of cortex in a Thy1-YFP line $\mathrm{H}$ mouse. Image courtesy of M. Levene, Cornell University, USA. have developed a technique that allows microscopes to peer deeper inside the brain, but that is no more invasive than an electrode.

The new method relies on gradient index (GRIN) lenses - needle-shaped lenses that use a negative gradient in the refractive index of glass from the centre of the lens to the edge. This gradient bends and focuses the light so that an image can be carried within the lens for several centimetres. The GRIN lens is inserted through a hole in the skull and dura, and allows fluorescence microscopy of tissue a few tens of micrometres from the end of the lens. By advancing the lens in stages, with pauses between each step, the authors hoped to minimize both tissue damage and tissue compression.

To test the usefulness of the technique, Levene and colleagues injected fluorescent quantum dots or fluorescein-dextran into mice, and then carried out microangiography of cerebral capillaries and larger blood vessels as deep as $2 \mathrm{~mm}$ below the surface of the brain. They also used transgenic mice that express yellow fluorescent protein in cortical and hippocampal neurons to view neurons about $1.5 \mathrm{~mm}$ below the surface with resolution high enough to view individual neurites.

The ability to view microscopic structures relatively deep within the living brain has great potential both for basic research and for clinical investigation. In this study, damage caused by insertion of the lens was minimal, although much more work will be needed to ensure that the technique can be safely used in humans. In the meantime, development of this technique to include the use of other labels or intrinsic signals should mean that it finds many uses in the laboratory.

Rachel Jones

\section{(2) References and links}

ORIGINAL RESEARCH PAPER Levene, M. J. et al. In vivo

multiphoton microscopy of deep brain tissue. J. Neurophysiol.

10 December 2003 (10.1152/jn.01007.2003)

FURTHER READING Helmchen, F. \& Denk, W. New

developments in multiphoton microscopy. Curr. Opin. Neurobiol. 12, 593-601 (2002) 